\title{
How to test the "quantumness" of a quantum computer?
}

\author{
Alexandre M. Zagoskin ${ }^{1,2 *}$, Evgeni I''ichev ${ }^{3}$, Miroslav Grajcar ${ }^{4}$, Joseph J. Betouras ${ }^{1}$ and Franco Nori ${ }^{2,5}$ \\ ${ }^{1}$ Department of Physics, Loughborough University, Loughborough, UK \\ ${ }^{2}$ Center for Emergent Matter Science, RIKEN, Saitama, Japan \\ ${ }^{3}$ Quantum Detection, Leibnitz Institute of Photonic Technology, Jena, Germany \\ ${ }^{4}$ Department of Experimental Physics, Comenius University, Bratislava, Slovakia \\ ${ }^{5}$ Department of Physics, University of Michigan, Ann Arbor, MI, USA
}

\author{
Edited by: \\ Jacob Biamonte, ISI Foundation, \\ Italy \\ Reviewed by: \\ Vasileios Basios, Université Libre de \\ Bruxelles, Belgium \\ James Daniel Whitfield, Vienna \\ Center for Quantum Science and \\ Technology, Austria \\ José Geraldo Peixoto De Faria, \\ Centro Federal de Educação \\ Tecnológica de Minas Gerais, Brazil \\ ${ }^{*}$ Correspondence: \\ Alexandre M. Zagoskin, Department \\ of Physics, Loughborough \\ University, Loughborough LE11 3TU, \\ UK \\ e-mail: a.zagoskin@lboro.ac.uk
}

Recent devices, using hundreds of superconducting quantum bits, claim to perform quantum computing. However, it is not an easy task to determine and quantify the degree of quantum coherence and control used by these devices. Namely, it is a difficult task to know with certainty whether or not a given device (e.g., the D-Wave One or D-Wave Two) is a quantum computer. Such a verification of quantum computing would be more accessible if we already had some kind of working quantum computer, to be able to compare the outputs of these various computing devices. Moreover, the verification process itself could strongly depend on whether the tested device is a standard (gate-based) or, e.g., an adiabatic quantum computer. Here we do not propose a technical solution to this quantum-computing "verification problem," but rather outline the problem in a way which would help both specialists and non-experts to see the scale of this difficult task, and indicate some possible paths toward its solution.

Keywords: quantum computing, adiabatic quantum computing, quantum coherence, quantum annealing, D-Wave Systems, quantum simulations, quantum speed-up
Suppose we are given a black box and told that this is a quantum computer. How do we test if it is capable of performing quantum computing? The standard approach would be to run a series of tests of few-qubit operations, and compare the outputs with results obtained using a standard (classical) computer simulating the quantum evolution. This is possible for small-size systems, likely comprising less than 40-50 qubits, given the current capabilities and fundamental limitations of classical computers (due to the exponential growth of computing resources needed to model large quantum systems [11]. But what if the system size is larger? Another challenging task would be to find out how to change the design of the "black-box computer" to improve its performance. Namely, if the black box computer is not working as a quantum computer, how to find this out and how to fix the problem?

Now we are facing a similar situation. The development of an "adiabatic quantum annealer" by D- Wave Systems Inc. over the last few years did produce reactions ranging from excitement to skepticism [2-9]. Considerable interest was generated by:

(1) the demonstration of quantum annealing in an 8-qubit register of the prototype processor [10];

(2) the sale of a 128-qubit quantum annealer "D-Wave One" to Lockheed Martin and its installation at the University of Southern California (2011), and its upgrade to a 512-qubit "D-Wave Two" (2013);

(3) the subsequent evidence of quantum annealing in the working 108 qubits of this device $[11,12]$;

(4) the realization of a quantum adiabatic algorithm on a nominally 512-qubit (439 qubits operational) device "D-Wave Two" outperforming at least some classical algorithms [13] and losing to some other classical algorithms [14];
(5) the May 2013 decision by NASA, Google and the Universities Space Research Association to purchase a "D-Wave Two" to be installed at their common Quantum Artificial Intelligence Lab at NASA's Ames Research Centre [15];

(6) the evaluation of small Ramsey numbers using the "D-Wave One" device [16].

Questions were raised concerning the relative speed of the D-Wave processors compared to classical optimization algorithms [7] and the quantum character of their evolution (see [17], the response [18] and the reply to the response [19]). While the former question is crucial for information science, the latter one is more important from the point of view of physics. We believe that resolving it would drastically improve the current status of the field, which we find unsatisfactory in several important respects. The very fundamental impossibility of an efficient simulation, with classical means, of large enough quantum systems [1], which provides the justification for the quest for quantum computing, may prevent us from developing quantum computers, unless better classical approaches toward their design and evaluation are found.

\section{CURRENT SITUATION}

The device ("D-Wave One"), tested in Boixo et al. [11, 12], consisted of 128 (108 of which were operational) $\mathrm{Nb}$ - based superconducting flux qubits (see, e.g., [20], Ch.2), arranged in blocks of 8 , with selective tunable couplings. This particular design used dc SQUIDs in place of Josephson junctions, which allowed to fine-tune the qubit parameters by changing the magnetic fluxes through the control loops. The amplitude and sign of the couplings between the qubits could also be tuned in a similar way 
[21]. The qubits formed a lattice, which can be modeled by a network of Ising spins with randomly chosen interactions.

The goal of the experiment $[11,12]$ was to find the statistics of the device by determining the random spin glass ground state and comparing the results with the algorithms based on simulated classical and quantum annealing (SCA and SQA, respectively). The surprising result was that the operation of D-Wave One produced a bimodal statistical distribution of success probabilities, corresponding to clearly distinct groups of "easy" and "hard" problems, similar to the one produced by SQA, but drastically different from SCA. In addition, they detected strong positive correlations of the success probabilities at different instances between the D-Wave One processor and SQA. As pointed out in Smolin and Smith [17], the bimodality itself was not sufficient to claim the evidence for quantum annealing, as it could be reproduced by semi-classical spin models. Nevertheless, the absence of correlations between semi-classical models and D-Wave One made a strong case in favor of quantum behavior, demonstrating some essential features of quantum annealing [11, 12, 18]. The above results are surprising, since the adiabatic evolution time of the processor $(5-15 \mu \mathrm{s})$ greatly exceeded the decoherence time of each separate qubit $(\sim 100 \mathrm{~ns})$ and therefore of the processor as a whole. More recent data obtained on a larger D-Wave Two processor seems to indicate the quantum character of the evolution [22] and show an improvement due to the use of a quantum error correction scheme [23].

\section{QUANTUM COHERENCE AND ENTANGLEMENT}

While the critical importance of maintaining quantum coherence for gate-based quantum computing is firmly established, the question of its role for universal adiabatic quantum computing, and its more limited versions (such as quantum optimization or approximate adiabatic quantum computing) is being debated (see, e.g., [24, 25]). Quantum coherence is certainly necessary, but on what scale, and for how long?

There is a point of view, according to which the existence of entangled energy eigenstates is not only necessary, but it may even be sufficient, for at least a limited operation of an adiabatic quantum computer. This was guiding some early efforts in this field [26-28]. Due to the fact that the tested structures consisted of a small number of superconducting flux qubits, it was possible to establish the existence of such eigenstates by the direct modeling of the quantum evolution of the system, and its comparison with the experimental data was possible. But for a general system the question remains: multiqubit entanglement is certainly necessary, but on what scale, and for how long?

\section{SCALING WITH THE NUMBER OF QUBITS}

The situation becomes qualitatively different with the current and future generations of multiqubit processors. The simulation of the full 108-qubit system in the quantum limit was not attempted, and therefore a direct investigation of the role of entangled energy eigenstates could not be undertaken. (Difficulties of such simulation were already explicit in Johnson et al. [10], where only a single 8-qubit register was investigated.) It was only possible to make a conjecture [18] that the better correlations between the behavior of the D-Wave processor and the SQA (compared to semi-classical spin models) was due to the fact that entangled energy eigenstates were used in the SQA calculations, but not in the semiclassical models. The exact classical optimization algorithms used in Boixo et al. [11, 12], as well as generic approximate algorithms, will take impractically long time to run for 512 qubits. For the specific structure of the D-Wave One processor, it is expected that the simulated annealer algorithms (both classical and quantum) will scale exponentially (as $\exp [a \sqrt{N}]$ ) with the number $N$ of qubits, the same as exact solvers $[11,12]$. Therefore, it is likely that their usefulness for establishing the quantum character of their evolution in the planned devices, with several thousand qubits, is questionable. However, since SQA does not simulate the actual quantum behavior of the system, its results are of questionable relevance for the prediction of the ability of a given large-scale quantum qubit array to demonstrate quantum behavior at a given level of environmental and intrinsic decoherence, dispersion of parameters, etc.

\section{THE PROBLEM AND POSSIBLE SOLUTIONS}

The looming impossibility to predict the behavior of any big enough quantum processor (adiabatic, gate-based, etc) and even to test it for "quantumness" using classical tools, is the elephant in the room, and it may effectively restrict any further progress. Even taking the optimistic view, that quantum computing is not fundamentally restricted (by, e.g., limits on the size of systems capable of demonstrating quantum behavior [29]), it is realistic to expect, based on the current state of art, that a quantum processor capable of simulating itself accurately and quickly enough to be useful, will contain significantly more qubits than the current or prospective $\mathrm{D}$-Wave machines.

A recent analysis of the perspectives of superconducting circuits as a platform for universal quantum computation [30] stressed the very high price of implementing quantum error correction (necessary for the gate-based quantum computing) or "surface code" quantum computing [31], which runs to hundreds or thousands of physical qubits per a logical qubit. Devoret and Schoelkopf [30] speculates that large quantum processors should perhaps rely on a modular approach (when the operation and functionality of unit modules can be separately tested and characterized), or on some "hardware-specific shortcuts" (like using non-linear oscillators instead of qubits as a basis for superconducting quantum computing). However, these speculations may be overly optimistic. In order to use quantum parallelism one should entangle a few dozen logical qubits, which is only possible if all, or a significant fraction, of the unit elements are in a quantum coherent state for some minimum period of time. It was recently demonstrated experimentally [32], that the quantumness of a gate-based quantum computer can be verified using a smaller quantum device. It would be very interesting to know whether this approach can be extended to quantum annealers or used to estimate the performance of such devices. But so far, we cannot avoid the need to estimate and evaluate the behavior of large, essentially quantum systems with classical means, in order to develop a useful quantum computer.

The problem pertains for quantum annealers, universal quantum computers (gate-based or otherwise), adiabatic quantum computers, quantum simulators, and even for simpler artificial 
quantum structures, such as quantum metamaterials, whose properties are essentially determined by quantum correlations and entanglement within large collections of artificial atoms interacting with the electromagnetic field.

Nevertheless, we believe that this problem can be solved. This will first require developing a better set of theoretical tools. A system of qubits is, after all, a quantum many-body system, which may be amenable to the approaches which worked so well in many applications to condensed matter physics and statistical mechanics. If, for the moment, we restrict the field to quantum annealers, then the existing theoretical formalism must be extended to efficiently include the two essential features of this problem: its essentially non-equilibrium, transitional character, and the importance of quantum coherence (e.g., following [33]). It would be desirable to have an efficient method of establishing the probability, that the observed set of runs of a large-enough quantum annealer cannot be reduced to classical physics, and of estimating the performance of such a device based solely on the device parameters. This would not require to classically simulate a particular run of the device and could use an appropriate set of variables characterizing the system at the macroscopic level, e.g., its non-trivial adiabatic invariants.

Such set of theoretical tools will be useful not just for applications to quantum computing, but in a wider field of quantum engineering and second-generation quantum technologies [34]. Moreover, it may prove indispensable when addressing problems such as quantum simulations $[35,36]$, quantum-to-classical transition in open systems [37] and the challenging new field of quantum biology [38]. However, the whole area cannot develop until the proper guidelines are obtained on what kind of systems and under what conditions macroscopic quantum behavior is likely to be realized, and what should be the signs of this realization. How to evaluate the "quantumness" of a black box is a challenging problem, which requires a concerted approach, appropriate for such a fundamental task.

\section{ACKNOWLEDGMENTS}

Evgeni Il'ichev acknowledges support from the EU 7th Framework Programme collaborative project iQIT. Miroslav Grajcar was supported by the Slovak Research and Development Agency under the contracts APVV-0515-10, DO7RP-0032-11, and European Community's Seventh Framework Programme (FP7/2007-2013) under Grant No. 270843(iQIT). Joseph J. Betouras was supported by EPSRC through grant EP/H049797/1. Franco Nori is partially supported by the RIKEN iTHES Project, MURI Center for Dynamic Magneto-Optics, and a Grant-in-Aid for Scientific Research (S).

\section{REFERENCES}

1. Feynman R. Simulating physics with computers. Int J Theor Phys. (1982) 21:467. doi: 10.1007/BF02650179

2. Hardy Q. Google buys a quantum computer. New York Times (2013 8 May).

3. Jones N. D-Wave's Quantum Computer Courts Controversy. Available online at: http://www.scientificamerican.com/article/d-waves-quantum-computercourts-controversy/ (2013 19 June).

4. Cartwright J. Has a quantum computer solved the 'party problem'? Physicsworld.com. (2013 September).

5. Smith G, and Smolin J. Viewpoint: putting "Quantumness" to the test. Physics (2013) 6:105. doi: 10.1103/Physics.6.105
6. Guizzo E. "Loser: D-wave does not quantum compute," in IEEE Spectrum (2009 December).

7. Aaronson S. D-wave: Truth Finally Starts to Emerge. (Blog Entry). Available online at: http://www.scottaaronson.com/blog/?p=1400 (2013).

8. Aron J. Google's Quantum Computer Flunks Landmark Speed Test. Available online at: http://www.newscientist.com/article/dn24882-googles-quantumcomputer-flunks-landmark-speed-test.html?full=true\#.UvF28fvurvY(2014 January).

9. Grossman L. The quantum quest for a revolutionary computer. Time 28 (2014 February).

10. Johnson M, Amin M, Gildert S, Lanting T, Hamze F, Dickson N. et al. Quantum annealing with manufactured spins. Nature (2011) 473:194. doi: 10.1038/nature10012

11. Boixo S, Albash T, Spedalieri F, Chancellor N, Lidar D. Experimental signature of programmable quantum annealing. Nat Comm. (2013) 4:3067. doi: $10.1038 /$ ncomms3067

12. Boixo S, Roennow T, Isakov S, Wang Z, Wecker D, Lidar D, et al. Evidence for quantum annealing with more than one hundred qubits. Nat Phys. (2014) 10:218. doi: 10.1038/nphys 2900

13. McGeoch $\mathrm{C}$, Wang C. "Experimental evaluation of an adiabiatic quantum system for combinatorial optimization," in CF'13. Ischia (2013 May 14-16).

14. Roennow T, Wang Z, Job J, Boixo S, Isakov S, Wecker D, et al. Defining and detecting quantum speedup. arXiv:1401.2910 (2014).

15. Johnston H. D-Wave sells second quantum computer - this time to NASA. Phys World (2013) 26:9.

16. Bian Z, Chudak F, Macready W, Clark L, Gaitan F. Experimental determination of ramsey numbers. Phys Rev Lett. (2013) 111:130505. doi: 10.1103/PhysRevLett.111.130505

17. Smolin J, Smith G. Classical signature of quantum annealing. arXiv:1305.4904 (2013).

18. Wang L, Roennow T, Boixo S, Isakov S, Wang Z, Wecker D, et al. Comment on: "Classical signature of quantum annealing." arXiv:1305.5837 (2013).

19. Shin S, Smith G, Smolin J, Vazirani U. How "Quantum" is the D-Wave machine? arXiv:1401.7087 (2014).

20. Zagoskin A. Quantum Engineering: Theory and Design of Quantum Coherent Structures. Cambridge: Cambridge University Press (2011). doi: 10.1017/CBO9780511844157

21. Harris R, Johansson J, Berkley A, Johnson M, Lanting T, Han S, et al. Experimental demonstration of a robust and scalable flux qubit. Phys Rev B (2010) 81:134510. doi: 10.1103/PhysRevB.81.134510

22. Vinci W, Albash T, Mishra A, Warburton P, Lidar D. Distinguishing classical and quantum models for the D-Wave device. arXiv:1403.4228 (2014).

23. Pudenz K, Albash T, Lidar D. Error-corrected quantum annealing with hundreds of qubits. Nat Comm. (2014) 5:3243. doi: 10.1038/ ncomms 4243

24. Sarandy M, Lidar D. Adiabatic quantum computation in open systems. Phys Rev Lett. (2005) 95:250503. doi: 10.1103/PhysRevLett.95.250503

25. Amin M, Truncik C, Averin D. Role of single-qubit decoherence time in adiabatic quantum computation. Phys Rev A (2009) 80:022303. doi: 10.1103/PhysRevA.80.022303

26. Izmalkov A, Grajcar M, Il'ichev E, Wagner T, Meyer HG, Smirnov A. et al. Evidence for entangled states of two coupled flux qubits. Phys Rev Lett. (2004) 93:049902. doi: 10.1103/PhysRevLett.93.049902

27. Grajcar M, Izmalkov A, Il'ichev E. Possible implementation of adiabatic quantum algorithm with superconducting flux qubits. Phys Rev B (2005) 71:144501. doi: 10.1103/PhysRevB.71.144501

28. van der Ploeg S, Izmalkov A, Grajcar M, Huebner U, Linzen S, Uchaikin ST, et al. Adiabatic quantum computation with flux qubits, first experimental results. IEEE Trans Appl Supercond. (2007) 17:113. doi: 10.1109/TASC. 2007.898156

29. Penrose R. The Emperor's New Mind. Oxford: Oxford University Press (1999).

30. Devoret M, Schoelkopf, R. superconducting circuits for quantum information: an outlook. Science (2013) 339:1169. doi: 10.1126/science.1231930

31. Fowler A, Mariantoni M, Martinis J, Cleland A. Surface codes: towards practical large-scale quantum computation. Phys Rev A (2012) 2012:032324. doi: 10.1103/PhysRevA.86.032324

32. Barz S, Fitzsimons J, Kashefi E, Walther P. Experimental verification of quantum computation. Nat Phys. (2013) 9:727. doi: 10.1038/nphys2763 
33. Zagoskin A, Savel'ev S, Nori F. Modeling an adiabatic quantum computer via an exact map to a gas of particles. Phys Rev Lett. (2007) 98:120503. doi: 10.1103/PhysRevLett.98.120503

34. Georgescu I, Nori F. Quantum technologies: an old new story. Physics World 17 (2012).

35. Wecker D, Bauer B, Clark B, Hastings M, Troyer M. Can quantum chemistry be performed on a small quantum computer? arXiv:1312.1695 (2013).

36. Georgescu I, Ashhab S, Nori F. Quantum simulation. Rev Mod Phys. (2014) 86:153. doi: 10.1103/RevModPhys.86.153

37. Schlosshauer M. Decoherence: and the Quantum-To-Classical Transition. Berlin-Heidelberg: Springer (2007).

38. Lambert N, Chen Y, Chen Y, Li C, Chen G, Nori, F. Quantum biology. Nat Phys. (2013) 9:10. doi: 10.1038/nphys2474

Conflict of Interest Statement: Alexandre M. Zagoskin is a co-founder and shareholder of D-Wave Systems Inc. Evgeni Il'ichev and Miroslav Grajcar were collaborating with D-Wave Systems Inc. in the past. Neither of them collaborated with D-Wave Systems Inc. after 2005. The authors declare that the research was conducted in the absence of any commercial or financial relationships that could be construed as a potential conflict of interest.

Received: 18 February 2014; paper pending published: 23 March 2014; accepted: 09 May 2014; published online: 30 May 2014.

Citation: Zagoskin AM, Il'ichev E, Grajcar M, Betouras JJ and Nori F (2014) How to test the "quantumness" of a quantum computer? Front. Physics 2:33. doi: 10.3389/ fphy.2014.00033

This article was submitted to Interdisciplinary Physics, a section of the journal Frontiers in Physics.

Copyright (C) 2014 Zagoskin, Il'ichev, Grajcar, Betouras and Nori. This is an openaccess article distributed under the terms of the Creative Commons Attribution License (CC BY). The use, distribution or reproduction in other forums is permitted, provided the original author(s) or licensor are credited and that the original publication in this journal is cited, in accordance with accepted academic practice. No use, distribution or reproduction is permitted which does not comply with these terms. 